\title{
La evaluación de los aprendizajes: un análisis sobre las modalidades aplicadas en la formación de profesores del Instituto de Profesores Artigas
}

\author{
The evaluation of the learning process; an \\ analysis of the modalities applied in the training of \\ teachers at the Instituto de Profesores Artigas
}

\begin{abstract}
Diana Shablico
Master en Educación, Universidad ORT Uruguay. Ingeniera Agrónoma, Universidad de la República. Profesora de Educación Media en la especialidad Ciencias Biológicas, Instituto de Profesores Artigas. Docente del Consejo de Formación en Educación y del Consejo de Enseñanza Secundaria.
\end{abstract}

Fecha de recibido: $17 / 02 / 2014$

Fecha de aceptación: 11/06/2014

\section{Resumen}

El presente trabajo contiene las líneas centrales de una investigación que tuvo como objetivo realizar un estudio sobre las modalidades de evaluación de los aprendizajes en la formación de docentes del Instituto de Profesores Artigas (IPA) en el área de las Ciencias Naturales (Biología, Química y Física).

La investigación constituyó un estudio de caso que se desarrolló mediante una metodología mixta cuantitativa y cualitativa de corte descriptivo y explicativo. Se utilizaron dos muestras constituidas por docentes y estudiantes, cuya variedad fue dada por la selección de integrantes de Biología, Química y Física, los diferentes niveles de cursos ( $1^{\circ}$ a $4^{\circ}$ año) y las distintas dimensiones del saber (asignaturas pedagógicas y disciplinares). La recolección de datos se llevó a cabo mediante encuestas a los estudiantes, entrevistas semiestructuradas a los docentes y análisis documental.

El análisis de las opiniones de los estudiantes constituyó una clara evidencia del predominio de una cultura de la evaluación centrada en la acreditación de los aprendizajes, con énfasis en la medición y la obtención de calificaciones.

Por su parte, el análisis de las perspectivas y pensamientos de los docentes formadores habilitó establecer una asociación entre la formación de grado y sus concepciones sobre la evaluación.

La valoración integrada de los datos y categorías construidas permitió interpretar que la evaluación de los aprendizajes transita por líneas tradicionales de orientación cuantitativa, en especial en las materias disciplinares.

Estos hallazgos permiten sostener la idea de que es necesario continuar investigando sobre los modelos y las prácticas de evaluación de aprendizaje en educación superior, especialmente en el actual contexto de transición hacia una formación docente con carácter universitario.

PALABRAS CLAVE: evaluación, aprendizajes, modalidades, formación docente. 


\section{Abstract}

This paper is based on the main lines of research on learning assessment at the Instituto de Profesores Artigas (Artigas Institute of Teachers - IPA) in the area of natural sciences (Biology, Chemistry and Physics). The research was a Case Study conducted using a mixed methodology (quantitative and qualitative) of a descriptive and explanatory character. Two samples consisting of teachers and students respectively were used. The variety within said samples was given by the selection of members from the Biology, Chemistry and Physics' Plan of Studies, and from different levels (1 $1^{\text {st }}$ to $4^{\text {th }}$ years). Data collection was carried out through surveys to students, semi-structured interviews with teachers and documentary analysis.

An analysis into students' opinions clearly shows the predominance of evaluation practices focused on accreditation of learning, with emphasis on measurement and on earning grades.

On the other hand, the analysis of the teachers' viewpoints permitted the researcher to establish a link between the teachers' undergraduate education and their concept of evaluation.

The integrated data assessment and the categories constructed permitted to interpret that the evaluation of the learning processes currently follows the traditional line of quantitative orientation, especially in disciplinary subjects.

These findings support the need of deepening on the study of the models and practices of learning assessment in higher education, especially in the transition context to a university teacher training.

KEYWORDS: Assessment, learning, models, teacher training

\section{Introducción}

La evaluación de los aprendizajes con intención formativa no es sinónimo de medir ni calificar como tampoco de clasificar, examinar o aplicar pruebas. Estas actividades, sin embargo, en ocasiones suelen formar parte del quehacer evaluativo y dan cuenta de un amplio campo semántico en torno al tema, en el cual las diferencias radican en los recursos que se utilizan, los usos asignados y los fines a los que se sirven (Álvarez Méndez, 2001),

La evaluación, pues, resulta una actividad compleja por sustentarse en una diversidad de supuestos teóricos y de situaciones en la práctica diaria del aula que determinan diferentes modalidades a seguir.

Esta situación y otras, tales como la escasa producción de información que a nivel nacional existe sobre el tema, la reflexión que realizan algunos docentes sobre su práctica y el surgimiento de la nueva institucionalidad universitaria en la formación de profesores, establecen la necesidad de generar nuevos conocimientos y evidencia empírica que permitan comprender en profundidad las prácticas de evaluación académica, con el propósito de poder transformar y mejorar la calidad de la enseñanza en la formación de docentes.

En tal sentido algunas de las principales preguntas que guiaron este estudio fueron las siguientes: ¿Cómo evalúan actualmente los docentes del IPA los aprendizajes académicos? ¿Qué concepciones poseen los estudiantes y docentes de los profesorados de Biología, Física y Química, sobre la evaluación? ¿Qué factores inciden en la toma de decisiones de estos docentes al momento de planificar su evaluación? ¿Cuáles son las perspectivas de profesores y estudiantes en torno a la evaluación de los aprendizajes frente al nuevo marco institucional universitario? 


\section{Antecedentes y fundamentación teórica}

El presente capítulo expone diferentes opiniones y conceptualizaciones de diversos autores que han estudiado y profundizado en la evaluación de los aprendizajes como actividad inherente a todo acto educativo.

Se tendrá en cuenta la perspectiva didáctica y pedagógica de los aportes, por ser estos dos aspectos medulares en la reflexión sobre el tema y en relación a las nuevas propuestas de la agenda educativa.

En primer lugar, se presentan algunos aportes considerados clave para la comprensión del sentido de la evaluación integrado a la acción educativa. Posteriormente, a efectos de profundizar y comprender uno de los ejes centrales de la investigación se incluye discusión y conceptualización de los diferentes modelos de evaluación en educación.

\section{El sentido de la evaluación}

En la actualidad, la evaluación se encuentra en una línea de cambio. Tal como lo expresa Perrenoud (2008), hay una transformación que se inicia lenta pero que avanza. Las reformas educativas expresan voces que se alzan en función de un mayor interés y dedicación al tema.

Contrariamente, en la realidad del aula el docente tropieza, en ocasiones, con preocupaciones y vicisitudes causadas por circunstancias que lo obligan a centrar su atención en objetivos lejanos a los propósitos de la evaluación cualitativa y en favor de prácticas tradicionales, con énfasis en los resultados finales más que en los procesos de aprendizaje.

Esta dualidad hace reflexionar sobre el vínculo entre las formas de pensamiento y las formas de acción en el quehacer del docente y da cuenta del trayecto que existe entre lo que el docente cree que debe hacer y lo que en realidad hace en sus prácticas de aula.

La situación expresada pone de manifiesto que los modelos tradicionales de corte cuantitativo tienen vigencia actualmente. Sin embargo, a través de investigaciones y reflexiones es posible observar una tendencia innovadora, hacia un cambio centrado en modelos de evaluación alternativos que contemplan más al alumno y sus procesos de aprendizaje en los nuevos contextos (Perrenoud, 2008).

En acuerdo con lo señalado por Álvarez Méndez (2001) y Santos Guerra (1995), es tiempo de trabajar con afán y renovada convicción, y con la capacidad de habilitar y considerar a la evaluación cualitativa como una actividad integral de toda la práctica educativa, de gran para el docente. Esta riqueza se fundamenta en el potencial y la fuerza de la evaluación para estimar los procesos de aprendizaje de los estudiantes dado que permite interpretar sus necesidades, dificultades y fortalezas, como también otorga al profesor elementos para reflexionar y revisar su propia práctica.

Estas acciones logran concretarse cuando el docente posterga el énfasis puesto en los contenidos que enseña y comienza a centrar su actividad en la comprensión de los procesos que permiten a sus alumnos realizar aprendizajes significativos.

En la misma línea de pensamiento, las instancias aisladas de evaluación, como también las realizadas al término de una unidad o tema de carácter repetitivo, únicamente habilitan a una calificación que, si bien es necesaria, proporciona poca información sobre el proceso y puede representar medios para clasificar, seleccionar y excluir estudiantes.

En este sentido cabe destacar las coincidencias en los aportes de Álvarez Méndez (2001), Díaz Barriga (2000), Litwin (2005) y Santos Guerra (1998), quienes hacen énfasis en la importancia de reconsiderar en forma permanente los momentos oportunos para evaluar, en el entendido de que éstos deben ser estratégicos y utilizados como herramientas válidas para reconocer circunstancias especiales, obstáculos y aciertos.

En atención a lo expuesto, la evaluación es considerada un elemento central de la "nueva agenda de la didáctica" (Litwin, 2005, 11) si se considera que su acción se gesta en un ámbito reflexivo de clase y, por su intermedio, alumno y docente trabajan en forma colaborativa para la construcción de aprendizajes. 
Desde esta nueva visión, es importante que el docente posea una concepción clara del marco teórico que sustenta su evaluación en función de articularla y conjugarla en forma consistente y coherente con las teorías de enseñanza y aprendizaje que desarrolla en sus clases (Camilloni, 2005).

Los aportes realizados inscriben a la evaluación en una relación social entre actores que en la actualidad se encamina en una dirección de cambio. Pese a ello, aún se sitúa entre dos discursos, uno al servicio de la certificación y el otro al servicio de la formación. Del camino que se tome dependerá el sentido de la evaluación.

\section{Modelos de evaluación}

Existen dos lógicas del quehacer evaluativo: el modelo cuantitativo y el modelo cualitativo. Ambos constituyen verdaderos "paradigmas" (House 2000, 23) y como tales representan un conjunto de ideas que guían y orientan las formas de acción y toma de decisiones.

Los modelos cuantitativos de evaluación consideran la finalidad de los actos éticos en la realización de alguna acción individualista y utilitaria, en donde cualquier labor que logre ese objetivo es adecuada y debe estimularse. La evaluación bajo esta óptica se relaciona con los valores promedio de las pruebas o test. A partir de un producto final se busca un ordenamiento dentro de determinada escala con beneficios directos para el interesado. Se define de este modo el propósito de la evaluación cuantitativa, el cual gira en torno a la medición del rendimiento estudiantil a través de actividades objetivas y estandarizadas. Los resultados finales son elementos mensurables que habilitan el cotejo y la comparación (House 2000).

En los modelos cualitativos de evaluación prevalecen pautas que emanan de acuerdos plurales entre los integrantes de la sociedad. El debate y el diálogo constituyen el medio que posibilita el intercambio de opiniones y ponen de manifiesto diferentes intereses y costumbres en procura de un entendimiento genuino, capaz de otorgar un sistema de valoraciones más justo (House 2000).

La generación y validación del conocimiento es otra distinción entre los modelos. Desde la orientación cuantitativa los resultados obtenidos a partir de pruebas estandarizadas tipo test son analizados mediante técnicas estadísticas. Esta línea de pensamiento encuentra su sentido en el paradigma positivista con una visión netamente mecanicista y de uniformidad de la naturaleza.

Los modelos cualitativos conciben una realidad compleja delineada por diversas causas dinámicas. La evaluación se relaciona con sus protagonistas y con la experiencia de éstos en el contexto de referencia. El evaluador se encamina a la búsqueda de múltiples miradas, de manera de poder valorizar actitudes reflexivas y de cooperación que permitan la observación del conocimiento implícito más que el explicito. Asimismo su actividad se orienta a la promoción de la autonomía y a la valoración de los procesos más que a la obtención de resultados.

Los modelos analizados representan extremos opuestos del pensamiento epistemológico y desde los cuales es posible contemplar los fenómenos sociales bajo diferentes ópticas. A partir de ellos afloran distintas explicaciones y formas de acción que se concretan en los diversos trayectos curriculares. La evaluación desarrollada por el docente no es ajena a estas ideas. Por el contrario, su actividad da cuenta de la concepción que en él existe acerca de cómo se genera y valida el conocimiento y la educación en su conjunto. El conocer las bases epistemológicas de las posibles alternativas a desarrollar en el aula habilita al cuerpo docente a realizar un trabajo más reflexivo y coherente.

\section{Diseño metodológico}

Los objetivos enunciados para el trabajo en conjunto con las fuentes de información del marco conceptual orientaron la elección de la metodología y las técnicas a desarrollar. 


\section{Estudio de caso}

El presente trabajo de investigación se desarrolló en el Instituto de Profesores Artigas (IPA), constituyendo un Estudio de Caso, con el propósito de indagar y analizar en profundidad la evaluación de aprendizajes integrada a la dinámica educativa del Instituto, a través de variadas fuentes de evidencia (Stake, 1998).

El estudio se realizó mediante una orientación mixta o multi-método cualitativo-cuantitativo que encuentra sustento en sumar fortalezas y ampliar los recursos para el tratamiento holístico del fenómeno a estudiar (Denzin \& Lincoln, 2005). En tal sentido, la metodología utilizada permitió obtener evidencia empírica desde la perspectiva y concepciones de sus protagonistas, observar la realidad, determinar variables y establecer su relación de forma de conocer en profundidad el valor atribuido a la evaluación de los aprendizajes en el IPA.

\section{Determinación de la muestra Docentes}

Dentro del universo de análisis representado por los docentes del IPA se optó por realizar una muestra intencional compuesta por profesores que desempeñan sus actividades en el ámbito de las Ciencias Naturales, las que se encuentran conformadas por las especialidades de Física, Química y Biología. La elección sobre el grupo de ciencias a trabajar en la investigación se basó en el interés del investigador por profundizar en el campo disciplinar de su formación de grado.

A partir del relevamiento sobre el número total de docentes que se desempeñaron durante el año 2012 en Ciencias Naturales en el IPA, se estimó trabajar con catorce de los mismos. La muestra real de docentes se redujo a nueve por razones externas a la voluntad del investigador.

La muestra intencional docente se configuró con la estructura que expone la Tabla $N^{\circ} 1$.

\section{Tabla $\mathbf{N}^{\circ}$ 1. Número de docentes que conforman la muestra intencional}

\begin{tabular}{|c|c|c|c|c|c|c|c|c|c|c|c|c|c|c|c|c|}
\hline \multirow{2}{*}{\multicolumn{2}{|c|}{\begin{tabular}{|c|} 
Muestra \\
Nivel del curso
\end{tabular}}} & \multicolumn{4}{|c|}{ Biología } & \multirow[b]{2}{*}{ Total } & \multicolumn{4}{|c|}{ Física } & \multirow[b]{2}{*}{ Total } & \multicolumn{4}{|c|}{ Química } & \multirow[b]{2}{*}{ Total } \\
\hline & & $1^{\circ}$ & $2^{\circ}$ & $3^{\circ}$ & $4^{\circ}$ & & $1^{\circ}$ & $2^{\circ}$ & $3^{\circ}$ & $4^{\circ}$ & & $1^{\circ}$ & $2^{\circ}$ & $3^{\circ}$ & $4^{\circ}$ & \\
\hline \multirow{2}{*}{ 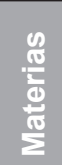 } & $\begin{array}{l}\text { Tronco } \\
\text { común }\end{array}$ & - & - & 1 & 1 & 2 & & & & & - & 1 & & & 1 & 2 \\
\hline & Especificas & - & - & 1 & 1 & 2 & & 1 & & 1 & 2 & $1^{*}$ & & $1^{*}$ & & 1 \\
\hline \multicolumn{2}{|r|}{ Totales } & & & & & 4 & & & & & 2 & & & & & 3 \\
\hline
\end{tabular}

Fuente: Elaboración propia

Referencias: * mismo docente, por lo que se cuenta como uno

\section{Estudiantes}

Los estudiantes conformaron otro componente de la muestra de trabajo. El universo de estudio comprendió todos los estudiantes inscriptos a cursar $3^{\circ}$ y $4^{\circ}$ año en las especialidades que contemplan las Ciencias de la Naturaleza, es decir, profesorados de Biología, Física y Química en los turnos matutino, intermedio y vespertino del IPA en el año 2012. El criterio de selección alude a que los estudiantes de estos niveles por su trayectoria formativa en la institución poseen experiencia en situaciones de evaluación de los aprendizajes en el IPA.

A partir de la disposición del universo, se estableció el marco de muestreo que se llevó adelante por medio de la consulta a los docentes entrevistados sobre el número de alumnos que concurren en forma real a sus clases al momento de realizar la encuesta. El mes determinado para tal fin fue setiembre de 2012 y la pesquisa arrojó un valor total de 87 estudiantes. Teniendo en cuenta este dato, se pensó en trabajar con la totalidad de alumnos. Sin embargo se logró aplicar la técnica seleccionada a 65 estudiantes, número que configuró la muestra final. 
La siguiente tabla $\mathrm{N}^{\circ} 2$ informa sobre el perfil y características de la muestra de alumnos encuestados.

Tabla $N^{\circ}$ 2. Perfil de estudiantes encuestados y conformación de muestra

\begin{tabular}{|c|c|c|c|c|c|}
\hline Especialidad & $\begin{array}{c}\text { Año de la } \\
\text { carrera }\end{array}$ & $\begin{array}{c}\text { Universo } \\
\text { real }\end{array}$ & Muestra & $\begin{array}{c}\text { Edad } \\
\text { promedio }\end{array}$ & $\begin{array}{c}\text { Año de ingreso } \\
\text { promedio a } \\
\text { la formación } \\
\text { docente }\end{array}$ \\
\hline \multirow{2}{*}{ Biología } & $3^{\circ}$ & 17 & 13 & 23 & 2009 \\
\cline { 2 - 6 } Química & $4^{\circ}$ & 20 & 14 & 26 & 2008 \\
\cline { 2 - 6 } & $3^{\circ}$ & 12 & 9 & 31 & 2009 \\
\hline \multirow{2}{*}{ Física } & $4^{\circ}$ & 16 & 14 & 26 & 2008 \\
\hline Totales & $3^{\circ}$ & 10 & 7 & 28 & 2009 \\
\hline
\end{tabular}

Fuente: Elaboración propia a partir de datos relevados

\section{Técnicas para la recolección de datos}

Las técnicas utilizadas fueron las siguientes:

\section{Entrevista semiestructurada a los docentes}

Para el análisis de la evaluación de los aprendizajes que realizan los docentes formadores se utilizó un proceso empírico que permitiera conocer la realidad y a tal fin se seleccionó la entrevista semiestructurada.

Mediante esta técnica se abarcaron las diversas dimensiones y particularidades de la evaluación de aprendizajes contemplados en los objetivos. Asimismo, se incluyeron otros aspectos interesantes que hacen al tema, como la coordinación de la evaluación y la transición hacia la vida universitaria.

El diseño de la entrevista se basó en un modelo flexible de veinte preguntas abiertas organizadas en tres ejes de valoración, a saber:

concepciones sobre evaluación

la evaluación de aula y parcial

el examen

\section{Encuesta a los alumnos}

A través de la utilización de esta técnica se recolectó información estructurada sobre opiniones, actitudes y creencias de los estudiantes en relación al tema evaluación. La elaboración del cuestionario tuvo presente los objetivos de la investigación y respetó una estructura lógica y coherente respecto del tema.

El diseño de la encuesta se estructuró al igual que la entrevista, mediante los tres ejes valorativos mencionados y se organizó a través de un primer apartado en relación al perfil del estudiante, una batería de preguntas abiertas que captaron formas de pensamiento y concepciones acerca de diversos aspectos de la evaluación y un grupo de preguntas cerradas con escala de respuestas vinculadas a la evaluación de los aprendizajes en el Instituto.

\section{Análisis documental}

La técnica se basó en el estudio de los documentos del Plan 1977 y del Plan 2008 -la normativa vigente para la formación de profesorado- por ser éstos relevantes en referencia al trabajo de investigación.

El análisis de la información aportada por esta técnica se realizó mediante la modalidad cualitativa de investigación desde la marco del paradigma constructivista. 


\section{Resultados y discusión}

Esta sección comprende el análisis de los datos que surgieron a partir del trabajo de campo y es producto de una actividad reflexiva que tomó como guía el marco teórico, los objetivos propuestos y las hipótesis sustantivas que guiaron la indagación.

Su organización se presenta en tres apartados a saber:

Análisis de Planes del IPA

La mirada del estudiante

La mirada del docente

La dinámica generada en los apartados de análisis, así como también la que resulta de la vinculación de las distintas interpretaciones fueron puntos de partida para construir nuevos significados que permitieron avanzar en el conocimiento sobre el tema.

\section{Análisis de Planes del Instituto de Profesores Artigas}

El análisis de los Planes 1977 y 2008 contribuyó con datos significativos sobre la visión epistemológica en torno al tema evaluación que resultaron ser elementos clave para la triangulación.

La reflexión sobre los documentos habilitó la construcción de los siguientes pensamientos.

\section{Plan 1977. La evaluación mecánica y competitiva}

El Plan pautó en forma meticulosa el accionar del educador y los programas a desarrollar. El perfil docente se alineó a un rol técnico, aplicador de propuestas ya implementadas para su ejecución y estimador de rendimientos. Desde esta mirada, la evaluación se afianzó en sus aspectos cuantitativos y fortaleció su condición utilitarista, competitiva y de arbitrariedad enmascarada bajo una figura de exactitud y equidad.

\section{Plan 2008. La evaluación innovadora}

El reflexionar sobre la normativa vigente permitió vislumbrar su orientación la cual supone un quiebre con las posturas más tradicionales. Su línea de acción considera una evaluación cualitativa, de carácter integral, reflexivo y comprensivo que forma parte del acto educativo y de la acción didáctica del docente en tanto informa sobre el desempeño del estudiante y permite su retroalimentación.

\section{La mirada del estudiante}

La percepción de los estudiantes acerca de la evaluación de sus aprendizajes constituyó una de las referencias centrales de este trabajo de investigación.

La naturaleza numérica de los datos relevados en las encuestas demandó la aplicación de metodología cuantitativa y la utilización de herramientas estadísticas.

La información obtenida se organizó en base a los tres ejes temáticos propuestos:

-Concepciones sobre evaluación

-Evaluación de aula y evaluación parcial

-El examen

Este abordaje permitió una comprensión ordenada y profunda del tema y posibilitó el progreso del estudio hacia niveles de mayor complejidad concretándose en un análisis asociativo.

\section{Primer eje temático. Concepciones sobre evaluación en el IPA}

Se consultó a los estudiantes sobre sus apreciaciones acerca de la evaluación de los aprendizajes en el IPA. A partir de las respuestas logradas y su procesamiento se construyeron categorías de análisis, las que se observan en el Tabla $\mathrm{N}^{\circ} 3$. 
Tabla $N^{\circ} 3$. Concepciones de los estudiantes de Biología, Química y Física de $3^{\circ}$ y $4^{\circ}$ año acerca del papel que desempeña la evaluación de aprendizajes en el IPA

\begin{tabular}{|l|c|c|}
\hline \multicolumn{1}{|c|}{$\begin{array}{c}\text { Respuestas originales más representativas } \\
\text { de los estudiantes }\end{array}$} & $\begin{array}{c}\text { Concepción sobre } \\
\text { evaluación }\end{array}$ & $\begin{array}{c}\text { Porcentaje } \\
\text { (\%) }\end{array}$ \\
\hline $\begin{array}{l}\text { "Filtro: qué estudiantes rendirán examen y cuáles no" } \\
\text { "Establecer la condición de exonerado, reglamentado } \\
\text { "Medir el rendimiento del alumno" } \\
\text { "Prepara para el examen" }\end{array}$ & $\begin{array}{c}\text { Evaluación } \\
\text { como acreditación } \\
\text { y medición de } \\
\text { saberes }\end{array}$ & 52,4 \\
\hline $\begin{array}{l}\text { "Control de contenidos" } \\
\text { "Se evalúa muchas veces lo memorístico" }\end{array}$ & $\begin{array}{c}\text { Evaluación como } \\
\text { reproducción } \\
\text { de contenidos } \\
\text { "Evetenidos en forma de contenidos" } \\
\text { memorística }\end{array}$ & 32,8 \\
\hline $\begin{array}{l}\text { "Evaluar conocimientos académicos puntuales" } \\
\text { "Formativa" a mín mismo" }\end{array}$ & $\begin{array}{c}\text { Evaluación } \\
\text { "Generar competencias" } \\
\text { "Autorregulación de conocioncia de }\end{array}$ & 14,8 \\
\hline
\end{tabular}

Fuente: Elaboración propia

Las dos primeras categorías, evaluación como acreditación y como reproducción de contenidos, pueden unificarse por converger en una concepción sobre evaluación más tradicional, de enfoque cuantitativo y conforma un $85,2 \%$ frente al $14,8 \%$ integrado por ideas más innovadoras de enfoque cualitativo.

El valor de $85,2 \%$ resulta ser un dato alto y significativo, puesto que de cada diez opiniones relevadas por lo menos ocho coinciden en que la evaluación de los aprendizajes en el instituto de formación se orienta por líneas cuantitativas.

Las apreciaciones de los estudiantes son producto de su experiencia personal, cuya construcción está sujeta a los trayectos formativos que han realizado y realizan, dado que al momento su educación se encuentra en más de la mitad o a término de la carrera, por cursar el tercer o cuarto año.

En este sentido es posible realizar el planteo acerca de qué circunstancias pueden determinar los resultados, teniendo presente que los alumnos transitaron y continúan su formación bajo la oferta curricular del Plan 2008, el cual explícitamente orienta la evaluación mediante un enfoque cualitativo.

\section{Segundo eje temático. Evaluación de aula y evaluación parcial}

El Plan 2008 establece la posibilidad de exoneración de las asignaturas a través de la valoración de los procesos de aprendizaje que el estudiante realiza durante el año. La consulta a los mismos sobre este aspecto brindó los resultados que muestra el Gráfico № 1. 


\section{Gráfico $N^{\circ}$ 1. Porcentaje de exoneración de asignaturas del tronco común y específicas}

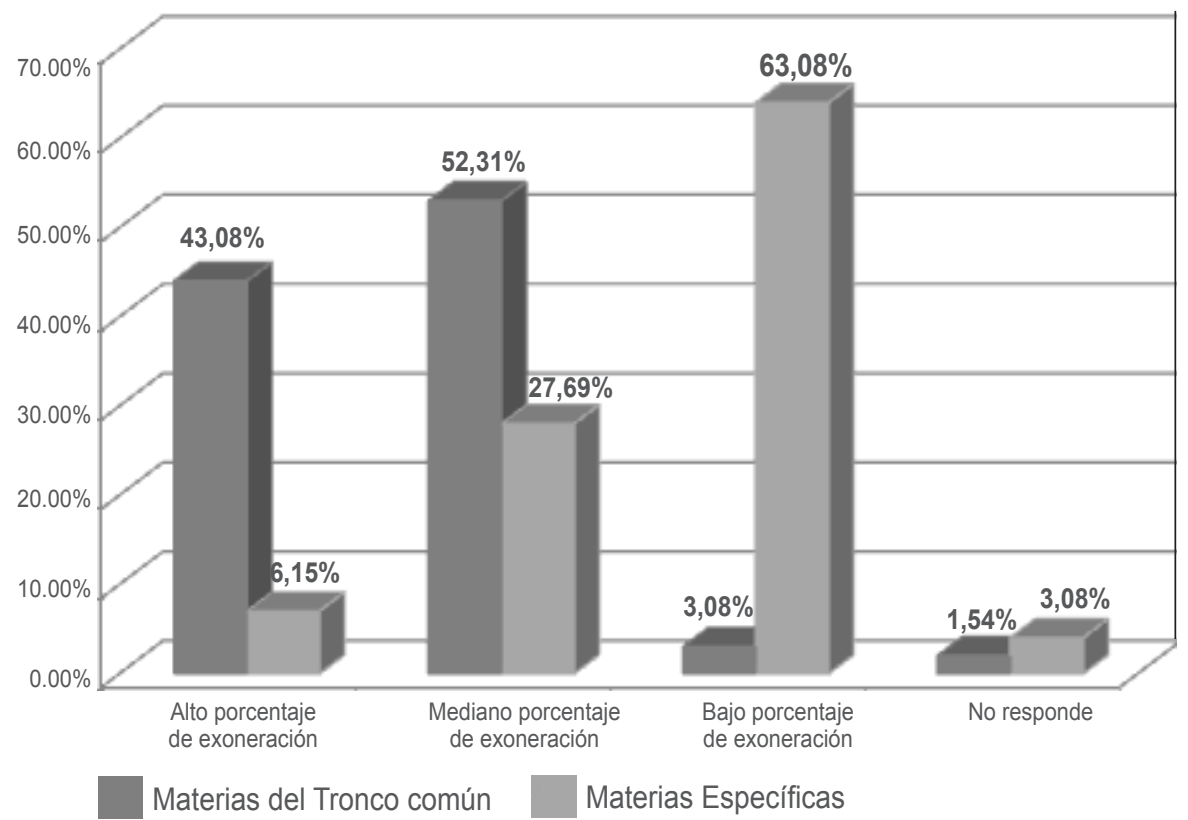

Del análisis del gráfico es posible observar dos claras tendencias con sentido opuesto:

- Las materias del tronco común presentan un alto a mediano porcentaje de exoneración puesto que más del $95 \%$ de los estudiantes concuerda en esta opinión.

- Las materias específicas muestran un bajo porcentaje de exoneración, dado que más del $63 \%$ de los encuestados coincide en este punto de vista.

En la búsqueda de elementos que aportaran mayor información se consultó a los estudiantes sobre la conformación de la nota final del curso y su determinación a través de las notas obtenidas en los parciales. Los resultados se muestran en el gráfico $\mathrm{N}^{\circ} 2$.

Gráfico $N^{\circ}$ 2. Valoración de los estudiantes sobre la incidencia de las notas parciales en la nota final del curso

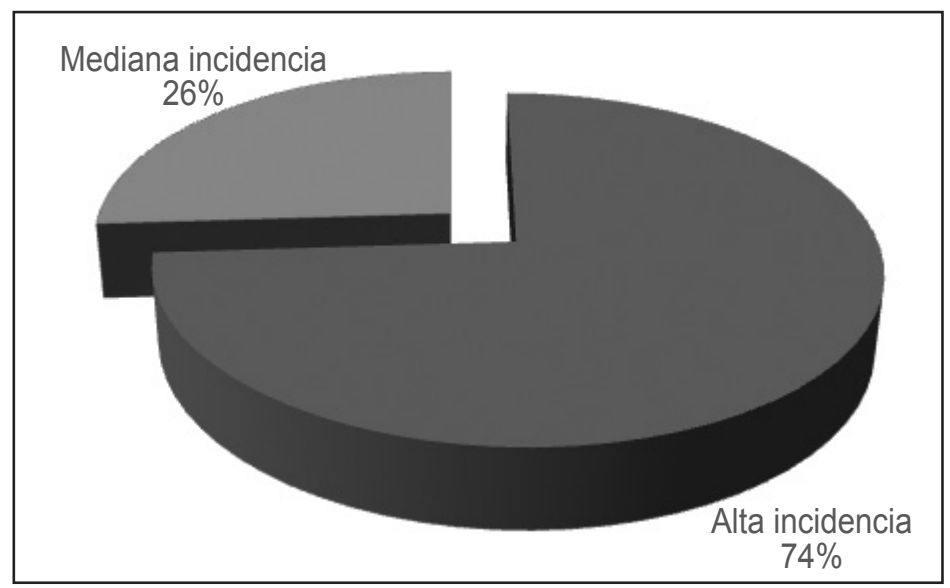


La información que surge de la imagen es categórica en el sentido de que la sumatoria de las opciones "mediana y alta incidencia" representan el $100 \%$ de las respuestas. Ningún estudiante encuestado marcó el ítem de "baja incidencia" que formaba parte de las opciones. Las notas de los parciales inciden mayoritariamente en la determinación de la nota del curso.

Esta apreciación de los estudiantes coincide con la idea de que la evaluación se conduce en mayor grado mediante formas estancas, al estilo de pruebas parciales y test en desmedro de una valoración dinámica de los procesos de aprendizaje. A partir de lo expresado cabe preguntar: el parcial como espacio de evaluación ¿se encuentra sobredimensionado desde la visión del docente?

Con el propósito de profundizar en esta idea se analizaron los datos resultantes acerca del tipo de preguntas que conforman las pruebas parciales. Los resultados se observan en el gráfico $\mathrm{N}^{\circ} 3$.

\section{Gráfico $\mathbf{N}^{\circ} 3$. Conformación de las propuestas parciales a través de valoraciones de los estudiantes}

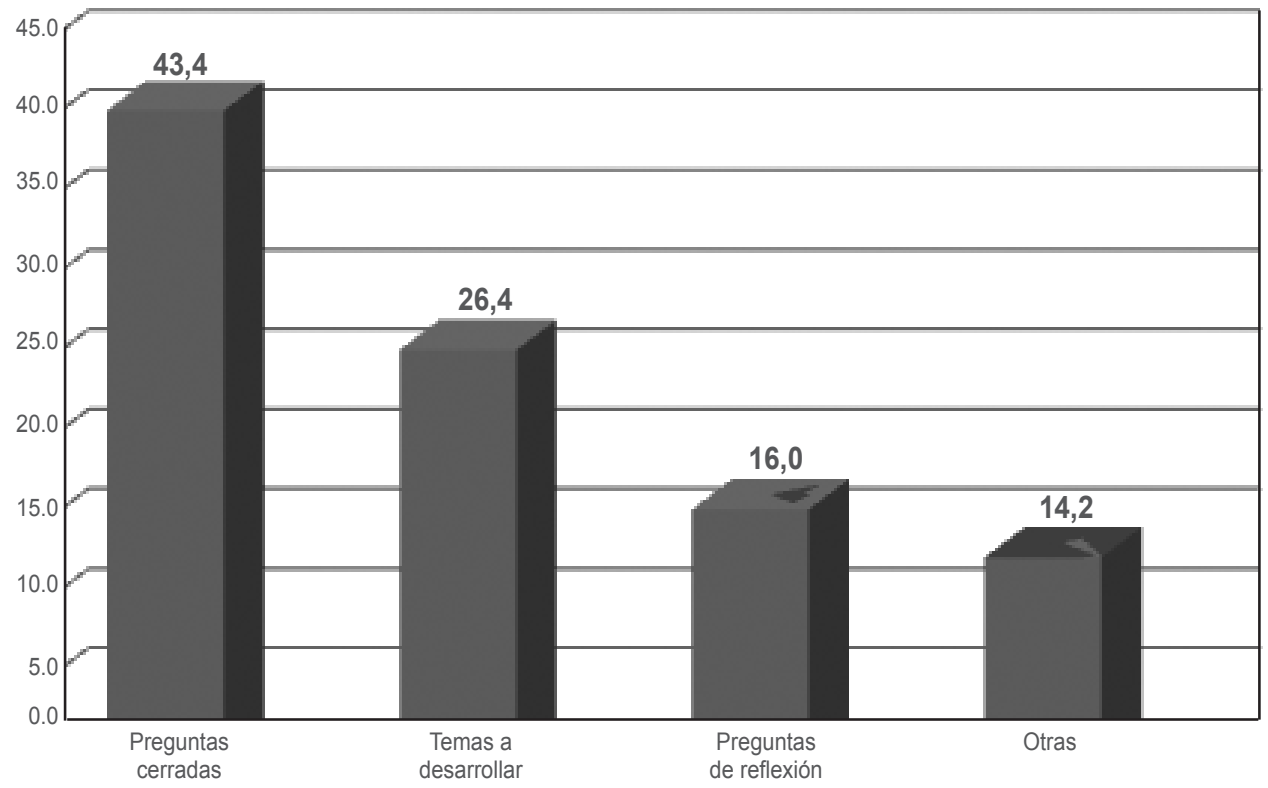

La representación gráfica pone de manifiesto que las propuestas en base a preguntas cerradas son la forma más utilizada en los parciales y coexisten con otro tipo de propuestas.

Su predominio y preferencia por parte de los docentes centraliza la evaluación en los resultados o la respuesta final. Tal como lo expresa Kincheloe (2001), las propuestas de evaluación con énfasis en interrogantes que convergen en respuestas correctas y únicas favorecen la fragmentación de contenidos y del pensamiento.

\section{Tercer eje temático. El examen}

Desde una perspectiva social e institucional, el examen acredita o certifica a los estudiantes respecto de la adquisición de conocimientos y los habilita a su pasaje de grado u obtención del título.

De este modo se consultó a los alumnos sobre el examen como instancia final de evaluación. Los resultados se presentan en el Tabla $\mathrm{N}^{\circ} 4$. 
Tabla $N^{\circ} 4$. Resultados de la consulta sobre el examen

\begin{tabular}{|c|c|c|}
\hline Opciones & $\begin{array}{c}\text { ¿Se evalúa el examen mediante } \\
\text { los mismos criterios que se } \\
\text { utilizan para evaluar el curso? }\end{array}$ & $\begin{array}{c}\text { En la calificación final del } \\
\text { examen ¿crees que se tiene } \\
\text { presente la calificación del } \\
\text { curso? }\end{array}$ \\
\hline Siempre & $9,23 \%$ & $3,08 \%$ \\
\hline Algunas veces & $70,77 \%$ & $24,62 \%$ \\
\hline Rara vez & $12,31 \%$ & $44,62 \%$ \\
\hline Nunca & $1,54 \%$ & $21,54 \%$ \\
\hline Respuesta en blanco & $6,15 \%$ & $6,15 \%$ \\
\hline
\end{tabular}

Fuente: Elaboración propia

Los resultados presentes en el cuadro expresan una marcada tendencia a conformar la nota final del examen con escasa o nula participación de la calificación del año. De igual forma, los criterios que guían esta evaluación no necesariamente coinciden con los del curso. Estas ideas reflejan al examen como una "mega" instancia, disociada de los procesos de enseñanza y aprendizaje que el estudiante realiza, con reglas y características propias que lo definen como un instrumento estático sobre el cual se depositan expectativas y se toman decisiones de aprobación o reprobación de los cursos.

Además de la información relevada en los tres ejes temáticos, se consultó a los estudiantes en relación al proceso de transición hacia la formación docente universitaria. En este sentido se indagó sobre la existencia de intercambios acerca de cómo debería ser la evaluación de los aprendizajes en el nuevo contexto. La información resultante se muestra en el siguiente gráfico.

Gráfico $N^{\circ}$ 4. Grado de intercambio entre estudiantes acerca del tema evaluación en la transición hacia la formación docente universitaria

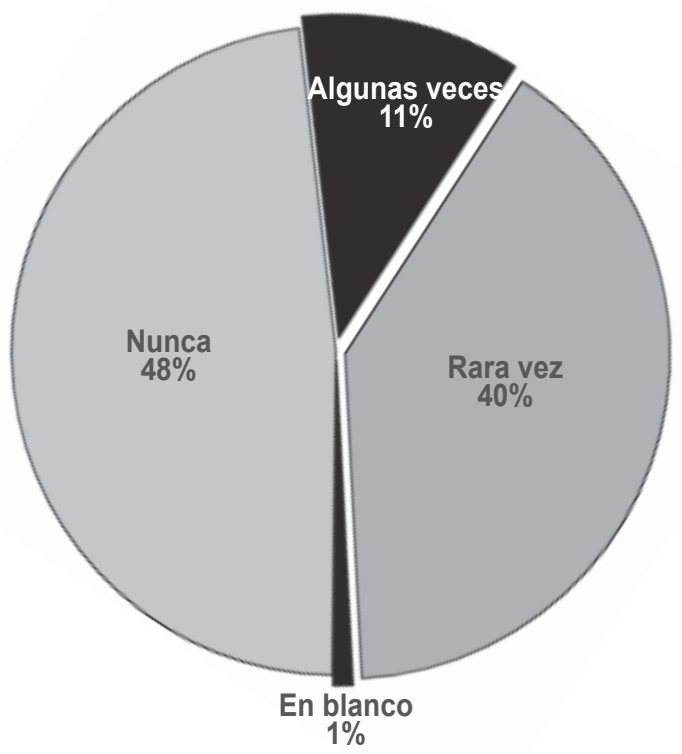


Las respuestas señalan un bajo grado de intercambio sobre la temática en cuestión. Los valores de $40 \%$ y $48 \%$ en las opciones "nunca" y "rara vez" respectivamente conducen a pensar que la evaluación de los aprendizajes en el marco de la nueva institucionalidad no reviste un interés de prioridad en los estudiantes. De esta forma es posible plantear nuevas interrogantes acerca de si el estudiante comprende el papel que desempeña la evaluación dentro de la educación.

\section{Análisis asociativo}

Con el propósito de profundizar en el análisis de los datos relevados se establecieron algunas hipótesis de trabajo y se buscó el grado de asociación entre las variables participantes. En este sentido se estudió la incidencia de las notas parciales en la calificación final del curso y su relación con las concepciones de los estudiantes sobre evaluación. Los datos resultantes se encuentran en la Tabla $\mathrm{N}^{\circ} 5$.

Tabla $\mathbf{N}^{\circ}$ 5. Concepciones de evaluación en los estudiantes según la incidencia de las notas de parciales en la calificación final del curso (\%)

\begin{tabular}{|c|c|c|c|c|}
\hline \multirow{2}{*}{\begin{tabular}{|c|} 
Concepción sobre \\
evaluación de aprendizajes \\
en estudiantes
\end{tabular}} & \multicolumn{3}{|c|}{$\begin{array}{l}\text { Incidencia de las notas de parciales en la } \\
\text { calificación final del curso }\end{array}$} & \multirow[b]{2}{*}{$\begin{array}{l}\text { Diferencia } \\
\text { porcentual }\end{array}$} \\
\hline & Alta & Media & Total general & \\
\hline Modelos cuantitativos & $90,6 \%$ & $61,8 \%$ & $83,1 \%$ & $28,8 \%$ \\
\hline Modelos cualitativos & $9,4 \%$ & $38,2 \%$ & $16,9 \%$ & $-28,8 \%$ \\
\hline Total general & $100 \%$ & $100 \%$ & $100 \%$ & \\
\hline
\end{tabular}

Fuente: Elaboración propia a partir de análisis de datos de encuesta

A partir del cuadro se observa que las concepciones de los estudiantes sobre la evaluación se encuentran en su mayoría alineadas a los enfoques cuantitativos (83,1\% versus $16,9 \%)$. Se conserva esta tendencia al relacionar ambas variables. En este sentido es posible apreciar que cuando el quehacer docente hace énfasis en las notas de las pruebas formales y, en suma, son las responsables de la calificación final del año, esto determina en los estudiantes un alto porcentaje de percepciones de evaluación en relación con el medir y acreditar (90,6\%).

En esta misma línea de análisis, una incidencia media de las notas de los parciales sobre los resultados finales de aprobación permite forjar en los estudiantes un pensamiento más holístico en torno a la evaluación, puesto que se incrementa el porcentaje de estudiantes a valores del 38,2\% con ideas fundadas en los modelos cualitativos. Esta situación es reflejo de la participación de otras actividades alternativas de evaluación durante el curso, que son tenidas en cuenta por estos estudiantes.

Se calculó el coeficiente de asociación Q de Yule de forma de establecer la fuerza entre las variables. El mismo ofreció un resultado de - 0,712, que se traduce en una importante relación entre la incidencia de las notas parciales en la calificación final y los modelos que orientan la evaluación 


\section{La evaluación como actividad sobredimensionada y vacía}

La presentación de los datos a través de gráficos y tablas expone ideas que motivan a reflexionar y profundizar en el análisis.

La mirada del estudiante deja en evidencia una definición clara sobre la evaluación como requisito formal que acredita saberes traducidos en logros académicos. El alumno de las especialidades consideradas se prepara para aprobar la asignatura o el examen. Su esfuerzo e interés se centran en las calificaciones en primera instancia antes que en los procesos cognitivos del aprendizaje.

Se instala de esta forma en las aulas de formación de los futuros docentes una cultura en la acreditación, que orienta y da sentido a la educación hacia aspectos de medición y reduce el acto de aprender a un almacenaje memorístico de información.

Las concepciones de los estudiantes en relación a los exámenes y parciales confirman lo expresado: existe una visión sobredimensionada y vacía de estos espacios de evaluación, con sustento en el modelo cuantitativo que guía y orienta mayoritariamente su acción educativa.

\section{La mirada del docente}

Toda acción educativa del docente refleja una lectura acerca del mundo que lo rodea, es decir, su labor representa la concepción de cómo se genera y transmite el conocimiento. Desde este lugar, las percepciones de los docentes sobre la evaluación de los aprendizajes constituyeron una pieza fundamental para la comprensión del sistema de evaluación en su conjunto.

El abordaje cualitativo de los relatos de entrevista requirió de un ir y venir entre los datos relevados, los objetivos y el marco teórico en permanente reflexión, de manera de poder franquear la narración descriptiva y expandir la información a través de los procesos sociales e interacciones que, como portadores de significado, acontecen en torno a la evaluación.

Tal como se procedió con los datos de los estudiantes, el análisis se basó en los tres ejes temáticos y permitió la construcción e interpretación de los hallazgos categoriales que fueron representados a través de metáforas.

\section{Primer eje temático. Concepciones sobre evaluación en el IPA}

\section{Los caminos de doble vía}

La entrevista consideró en una primera instancia recoger la opinión de los docentes sobre la evaluación de aprendizajes a partir de sus experiencias de trabajo en el Instituto de Profesores Artigas. Las ideas relevadas en las diversas narraciones se representan en la siguiente figura.

\section{Figura $N^{\circ}$ 1. Representación del pensamiento docente acerca de la evaluación}

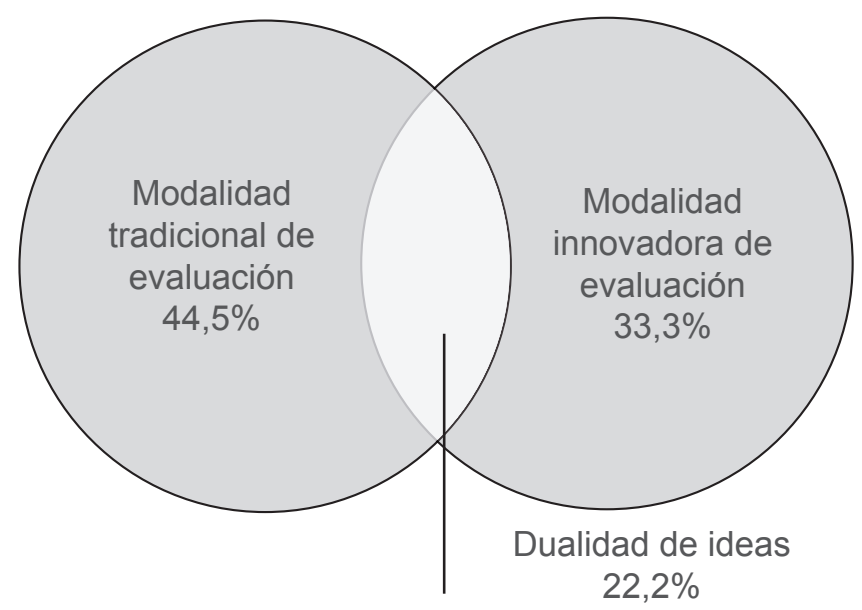

El esquema expresa dos modelos claros y definidos del pensamiento docente. 
Existió, a su vez, un grupo de profesores que trasmitió una dualidad de ideas. Sus discursos en un primer momento reflejan posturas transformadoras con sustento en la comprensión, que se desvanecen en el devenir de la entrevista y dan lugar a juicios y criterios conservadores.

La dualidad o yuxtaposición de ideas acerca de la evaluación son como caminos de doble vía que llevan o traen al docente, en este caso sin un rumbo muy fijo, y que ponen en relieve la complejidad que posee el tema, las múltiples concepciones que giran en torno a él, sus antagonismos y la escasa o nula reflexión que el docente realiza de sus acciones cotidianas en el aula.

\section{Tras la huella}

Los docentes de las asignaturas disciplinares expresaron pensamientos sobre la evaluación basados en modelos tradicionales con énfasis en la medición de respuestas concretas, precisas y sin errores.

El dominio de saberes, conceptos y procedimientos constituye una matriz para este grupo de profesores a través de la cual se crean, tal como lo expresa Perrenoud (2008), niveles jerárquicos de excelencia y se personifica a los estudiantes a partir de evaluaciones puntuales que generan su clasificación y selección. Se consagran en este sentido formas fijas e inamovibles de evaluación académica que se arraigan fuertemente y son consideradas tan naturales como imprescindibles por aquellas generaciones de docentes del IPA que "transitan por la misma huella". La aplicación masiva a través del tiempo les otorga reconocimiento y validez, convirtiendo a la acción de evaluar en ritos y costumbres integrados a la cultura de la evaluación impidiendo su transformación hacia formas flexibles y reflexivas.

\section{Una directriz de cambio}

La evaluación cualitativa se manifestó en las palabras, referidas a la dimensión pedagógica del Instituto, de varios docentes entrevistados que en general dictan clases en asignaturas del tronco común. Sus ideas expresan la importancia de valorar los procesos de aprendizaje de los estudiantes durante el curso por encima de las instancias puntuales de evaluación como son las pruebas formales.

La concepción sobre la evaluación de este grupo de docentes del IPA se despoja de su mandato medidor y pasa a ser una herramienta valiosa que permite reflexionar y comprender los procesos de aprendizaje. Los aspectos transformadores que se buscan por medio de su aplicación no son infalibles al igual que ocurre con otras estrategias. Sin embargo, es importante reconocer su capacidad para dar a la educación una identidad constructiva e integradora de propósitos.

\section{Historias repetidas}

El análisis interpretativo a partir de las opiniones de los docentes permitió indicar un fuerte vínculo entre los posibles caminos que toman la evaluación y el área de desempeño profesional. De esta forma, se constató que los docentes de materias específicas expresaron especial inclinación hacia enfoques de orientación cuantitativa, es decir, que en sus aulas desarrollan modelos tradicionales de evaluación.

Asimismo, los profesores que se desempeñan en materias que conforman el tronco común de aprendizajes se mostraron complacientes con los enfoques cualitativos, es decir, su quehacer está orientado hacia prácticas innovadoras, comprensivas y de reflexión con los estudiantes.

Esta información reviste mayor significación al tener presente otros aspectos que intervienen en el proceso educativo. En este sentido, aporta elementos de análisis el considerar los trayectos curriculares que fueron base de la formación profesional de los docentes entrevistados. 
Mediante este cruce de información se observó que, en su mayoría, los docentes de asignaturas disciplinares realizaron su formación de grado durante el Plan 1977 sustentado en la epistemología positivista.

Los docentes de asignaturas del área pedagógica responden a su vez a un período similar de formación en la Universidad de la República. La mayoría de ellos provienen de la Licenciatura en Ciencias de la Educación, en la cual la formación de grado implica una profundización en las diversas visiones epistémicas. A partir del conocimiento cabal, reflexivo y específico de que disponen, les resulta más sencillo alinearse a la postura de evaluación propuesta por el Plan 2008 y adaptarse al mismo tiempo a las bases del paradigma que lo sustenta.

En este sentido, la evaluación tradicional que se lleva a cabo en el Instituto de Profesores con base en la epistemología cuantitativa es aplicada principalmente en asignaturas disciplinares, volviéndose una "historia repetida" en la que se corre el riesgo de continuar perpetuando prácticas de aula y metodologías legitimadas en la formación de grado de los docentes a cargo de los cursos, formados a su vez en períodos de apogeo de la ideología positivista durante los cuales las respuestas medibles, exactas y el examen final tenían especial relevancia.

\section{Segundo eje temático. Evaluación de aula y evaluación parcial \\ Barreras al paso de nuevas pedagogías de evaluación}

La entrevista realizada a los docentes contempló la consulta acerca de los posibles factores que intervienen al momento de planificar la evaluación. Los temas con mayor presencia fueron el tiempo de que se dispone para elaborar y llevar adelante las instancias de evaluación, el tamaño de los grupos y su conformación. A partir de dichos conceptos afloraron aspectos tales como intereses, actitudes, compromiso y motivación.

Las opiniones recogidas en este apartado -como en otros- dan cuenta de que la evaluación no conforma un hecho educativo aislado. Por el contrario, se inscribe en una relación social particular y, por tal, es partícipe de las diversas situaciones por las que transitan el evaluador y el evaluado.

Los tiempos docentes, el alto número de alumnos que en ocasiones componen los grupos del IPA y la extensión de los programas dejan poco margen al quehacer educativo para pensar e implementar prácticas innovadoras de evaluación.

Según Perrenoud (2008), la evaluación con orientación formativa que intenta innovar consume mucho tiempo y esmero por parte del docente. Pese a ello, la acción didáctica se nutre y enriquece al permitir transformar a la práctica de evaluación en una dimensión del propio acto de enseñar, que proporcione información valiosa y habilite la comprensión y reflexión sobre las diversas situaciones del aula. De esta forma, evaluar implica comprender, negociar, establecer vínculos, tomar decisiones, actuar de manera reflexiva y constructiva. Frente a estos aspectos, los distintos factores expresados por los docentes intervienen a modo de "barreras" que impiden o dificultan la implementación de nuevas pedagogías abiertas y flexibles que habiliten la transformación de las prácticas de enseñanza.

\section{Tercer eje temático. El examen}

\section{Ritos y costumbres de la evaluación}

Las instancias formales de evaluación representaron un tema que se indagó a partir del análisis de las entrevistas realizadas a los profesores. La información obtenida reveló que los docentes de materias disciplinares prefieren elaborar pruebas en base a preguntas cerradas de opción múltiple, de desarrollo conceptual o ejercicios de única respuesta. En sus relatos se reconoce un denominador común representado por la exigencia de respuestas automáticas y repetitivas de conceptos teóricos que sólo dan cuenta de la receptividad, de la memoria del alumno y poco informan sobre el grado de construcción de los conceptos con orientación comprensiva y reflexiva. 
A partir de las opiniones de algunos profesores se percibe la idea del mayor nivel de exigencia o la mayor relevancia otorgada al examen como hecho natural y lógico, lo que da cuenta de la concepción sobredimensionada de este espacio de evaluación.

Por su parte, los docentes del área pedagógica manifestaron promover temas de análisis y reflexión en los cuales se reflejen argumentos, posturas e interpretación personal. Se establecen situaciones de evaluación que permiten comprender cómo sus estudiantes construyen sus conocimientos.

Los parciales y exámenes como instrumentos de evaluación formal del IPA cumplen funciones que pueden ser útiles desde el punto de vista educativo. Los posibles usos errados o equívocos de los mismos obedecen al tipo de saberes que con ellos se pretende explorar, a la clase de interrogantes que se formula para su construcción y a las exigencias que a partir de sus propuestas se demandan.

En el sistema actual de enseñanza parece imposible prescindir de estas configuraciones tradicionales de evaluación, fruto de rutinas y costumbres a lo largo del tiempo. Sin embargo, es factible pensar en su transformación hacia formas más legítimas de evaluar en las que se dé prioridad a la integración de saberes por encima de su exclusión, a la búsqueda y el entendimiento de los niveles de comprensión y a la contextualización de los procesos de enseñanza y aprendizaje.

\section{La calificación final del examen}

La mayoría de los docentes entienden razonable considerar el desempeño logrado en el año e integrarlo en la nota final del examen. Un porcentaje minoritario del grupo de docentes del área disciplinar se abstiene de hacerlo.

Si la nota final del examen no tiene en cuenta los procesos de aprendizaje que el estudiante realizó durante el año, el examen como tal es una evaluación de producto. Según Álvarez Méndez (2001), la actitud de desconocer la labor anual del alumno sitúa al examen como única instancia que define conocimientos. De esta forma se instala una concepción superficial del saber que reduce su existencia al triunfo obtenido en la prueba y magnifica a la vez su dimensión.

\section{La evaluación como ámbito privado}

En la entrevista a los profesores se consultó sobre la coordinación de la evaluación a nivel de las salas de docentes en las distintas áreas relevadas. Según lo manifestado por los profesores, en especial los de las materias pedagógicas, existe una coordinación entre algunos pares de asignatura donde se acuerdan los temas a abordar, la bibliografía, algunos criterios puntuales sobre las calificaciones y lo más relevante: las propuestas de exámenes surgen a partir de este ordenamiento en común del trabajo.

Si bien existen acuerdos previos para coordinar los procesos de enseñanza, no se advierte la necesidad de analizar las modalidades de evaluación alineadas a estrategias de enseñanza que orienten la construcción de saberes. La evaluación no se presenta como pensamiento central de los procesos educativos, lo cual denota que este tema no es prioridad de intercambio entre el colectivo docente.

El sistema tradicional de evaluación guía al docente por un riel sólido para transitar y estructurar en forma de rutinas el tiempo de clase en relación al tiempo de evaluar, así como también para relevar referencias que le permitan conocer si se progresa en la tarea.

En este marco operativo la evaluación no se discute, no se negocia, forma parte de un "ámbito privado", de un territorio no compartido del docente, y que depende en exclusividad de su elección y en consecuencia de sus creencias, concepciones y estimaciones personales. 
Según Perrenoud (2008) la evaluación que pretende innovar y formar no puede estar exenta del aporte colectivo y combinado de ideas con centro en los procesos de enseñanza y aprendizaje de los estudiantes, más allá de las individualidades del profesor. Los cambios, según este autor, en ocasiones son fuente de inestabilidad y nerviosismo por parte de los docentes porque crean situaciones que los alejan del "sólido riel" de la tradición y componen nuevos escenarios, ante los cuales los docentes son responsables.

\section{Una opción de cambio}

Durante la entrevista a los docentes también se relevaron las opiniones sobre la actual transición de la formación de profesores hacia modalidades de estructura universitaria. En este sentido, se consultó acerca de cómo debería implementarse la evaluación de aprendizajes en la futura formación.

Entre los datos recolectados surgen dos grandes tendencias de ideas al respecto: un grupo conformado en su mayoría por docentes de asignaturas disciplinares opinan que la evaluación de aprendizajes debe permanecer tal cual es en la actualidad por entender que es correcta y de buen funcionamiento. El otro grupo, conformado en su mayoría por docentes del área pedagógica, coincidieron en considerar a las propuestas de investigación y en especial las técnicas de observación y la entrevista como procesos adicionales e interesantes que permiten valorar cabalmente y en forma sostenida los aprendizajes de sus estudiantes.

A través de estas técnicas es posible tomar conciencia de la riqueza pedagógica y didáctica que de sus apreciaciones se desprende, ya sea porque permite al docente observar como integrante activo y reflexivo las distintas situaciones, o entrevistar a sus alumnos por medio de un diálogo horizontal, de respeto y mutuo entendimiento para valorar la solidez y coherencia de sus formas de razonar, sus avances y sus potencialidades cognitivas (Álvarez Méndez, 2001).

\section{Las diversas miradas}

La triangulación de los datos obtenidos a través de la aplicación de las técnicas permitió vincular los significados sobre el tema construidos por los docentes tanto con los registros aportados por los estudiantes como también con las directivas propuestas en la normativa vigente. Mediante esta estrategia se buscó contrastar la información relevada de manera de establecer un cuerpo coherente de ideas e incrementar la confianza en los hallazgos resultantes de la indagación.

El cruzamiento de los registros permitió analizar la discordancia entre las concepciones de evaluación entre ambos grupos. A través de las diversas expresiones y su codificación se pone de manifiesto que alumnos de docentes con enfoques innovadores poseen opiniones tradicionales de evaluación, con un cúmulo importante de conceptos que giran en torno a la acreditación del curso y a la evaluación de los contenidos. El resto de los estudiantes manifestaron posturas similares a las de sus educadores sustentadas en líneas cuantitativas.

A partir de estos resultados y profundizando en el análisis es factible establecer que los propósitos y representaciones innovadoras de los docentes, en acuerdo con la epistemología que los sustenta, constituyen piezas clave de los enfoques cualitativos de evaluación, siempre y cuando estén acompañados de la reflexión y la retroalimentación de la práctica docente. La ausencia de estos dos últimos aspectos podría ser una de las posibles causas de la discrepancia planteada entre estudiantes y docentes innovadores y da cuenta a su vez, tal como lo expresa Perrenoud (2008), de la brecha que existe entre lo que el docente se plantea hacer y lo que realmente hace en sus prácticas diarias. 
Por otra parte, el Plan 2008 del IPA -la oferta curricular vigente-, propone diversos principios y elementos que guían la propuesta evaluativa desde un enfoque cualitativo. Al triangular la propuesta del Plan con las concepciones sobre evaluación de los docentes y de los estudiantes se observó una divergencia significativa de ideas. Un porcentaje de alumnos superior al $80 \%$ expresó su opinión acerca de la evaluación de los aprendizajes como medición de saberes y acreditación, y más del $65 \%$ de los docentes manifestó su preferencia por la orientación de su labor mediante líneas tradicionales de evaluación.

Esta realidad divergente y de discrepancia entre las acciones cotidianas de evaluación de muchos docentes -en especial de aquellos de materias disciplinares- y lo que propone la normativa, se traduce en un escenario ambiguo para la formación de futuros profesores. Se refleja de esta forma disposición e interés por guiar a la evaluación a través de alternativas innovadoras sin tener presente o considerar las bases epistemológicas ni las certezas, convicciones, incentivos y costumbres de los profesores formadores. Este aspecto habilitó el repensar en los principales factores indicados por los profesores tales como los tiempos disponibles del docente durante el curso y el tamaño de los grupos del IPA, posibles obstáculos en la implementación de la evaluación cualitativa.

Estas ideas coinciden con el pensamiento realizado por Perrenoud (2008), quien establece que la evaluación con orientación innovadora determina situaciones que demandan tiempo y esfuerzo por parte del docente. Sin embargo, las instancias de retroalimentación a que dan lugar constituyen momentos valiosos que posibilitan la reflexión y aprendizaje de los estudiantes.

La calificación final del examen configuró otro punto de interés para el contraste de los datos. De éste surge que las opiniones de los estudiantes distan de las aportadas por sus profesores puesto que más del $65 \%$ opinó que "rara vez" o "nunca" se toman en consideración las notas del curso en la calificación final del examen.

Se ponen en relieve situaciones inconexas que están presentes en el ideario de los docentes y que sin embargo no son interpretadas de igual forma por sus estudiantes. Se establece una distancia entre las notas del curso y la calificación final del examen, lo que en ocasiones puede orientar la acción de los alumnos al desarrollo de estrategias de utilidad en relación con el saber para el logro de la certificación.

\section{Conclusiones}

En los diferentes apartados de este estudio se ha demostrado que el acto educativo es un proceso global en el cual la evaluación de los aprendizajes, que pretende ser formativa, se convierte en una dimensión del propio acto y de las situaciones didácticas. Desde esta perspectiva no es posible concebirla como una acción separada del proceso de enseñar y de aprender, así como tampoco puede considerarse desvinculada de las visiones epistemológicas y de los trayectos curriculares que enmarcan su actividad.

En función de los datos y las evidencias recogidos por esta investigación es posible realizar las siguientes conclusiones:

En la sección referida a los resultados de las opiniones de los estudiantes se destacaron diversos aportes y perspectivas que permitieron apreciar una concepción de la evaluación con base en la acreditación de los saberes con el fin de obtener logros académicos.

Esta visión restringida y estática determina una actuación y actitud de adiestramiento del alumno frente al aprendizaje en desmedro del valor intrínseco de los contenidos, el gusto por los mismos y los procesos que por su intermedio es posible desencadenar.

En los estudiantes, la cultura de la acreditación, con énfasis en la medición y la obtención de calificaciones, orienta los conceptos de "parcial" y "examen" transformándolos en instancias "sobredimensionadas y vacías" en las cuales convergen las distintas miradas y expectativas de los actores y que, como tales, poco aportan al proceso de aprendizaje genuino de los estudiantes 
y representan en muchos casos un conjunto de situaciones y medios para clasificar, seleccionar y excluir a los alumnos. Esta visión coincide con valoraciones y opiniones expresadas por algunos docentes, en especial aquellos dedicados a la dimensión disciplinar en el IPA puesto que otorgan un sobrevalor y relevancia a las calificaciones formales con un sentido utilitarista y se las desvincula de la evaluación de los procesos de enseñanza y aprendizaje.

Una de las principales conclusiones que surgen del análisis de las perspectivas y concepciones de los docentes es que la evaluación es considerada como un "agujero negro". La denominación de esta categoría emergente se incluye a partir del comentario de uno de los profesores entrevistados. Su valoración sobre la evaluación simbolizó el desconocimiento y desconcierto frente a las formas innovadoras de evaluación y asimismo sintetizó la mirada de varios docentes, específicamente de aquellos que se desempeñan en asignaturas disciplinares.

En efecto, el análisis interpretativo de las concepciones de los docentes permitió descubrir un fuerte vínculo entre los posibles caminos de la evaluación y el área de desempeño profesional. Los docentes de materias específicas se inclinan hacia enfoques tradicionales de evaluación centrados en los contenidos disciplinares y a través de los cuales se esperan respuestas fijas, exactas y que cuantifican el conocimiento. Por su parte los docentes de materias del tronco común de formación desarrollan en su mayoría acciones innovadoras en relación con las formas comprensivas y reflexivas en la evaluación de los aprendizajes.

Un hallazgo de este estudio fue el descubrimiento de una asociación entre la formación de grado de los docentes formadores y sus concepciones sobre la evaluación. Entre los docentes del área disciplinar se constató que mayoritariamente fueron formados en el marco del Plan 1977 -identificado claramente con ideologías tecnicistas de la evaluación-, mientras que los docentes abocados a la dimensión pedagógica del Instituto se formaron en su mayoría en la Facultad de Humanidades y Ciencias de la Educación con una profunda enseñanza en las distintas visiones epistemológicas del conocimiento.

La evaluación de los aprendizajes y sus modalidades dentro del proceso educativo no forman parte de un ámbito de coordinación participativa y de intercambio entre los profesores entrevistados del IPA. Esta situación genera un transitar en solitario del docente a partir del cual es casi imposible compartir experiencias y construir nuevas formas de ver el mundo.

El tiempo disponible para el ejercicio de la enseñanza, la extensión de los programas, el elevado número de alumnos que en ocasiones componen los grupos del IPA y sus particularidades específicas son, entre otros aspectos, los factores de mayor peso al momento de pensar la evaluación y constituyen, en muchas situaciones, obstáculos que impiden o dejan poco margen al quehacer educativo para idear e implementar prácticas innovadoras.

Los resultados obtenidos permiten confirmar que la transición hacia un nuevo escenario universitario de educación en la formación de profesores despierta poco interés tanto para docentes como para estudiantes. La búsqueda de formas adicionales de evaluación es considerada únicamente por parte de los profesores con visiones epistemológicas orientadas en base a modelos cualitativos. Los profesionales con enfoques tradicionales desisten de una idea de cambio en las formas de evaluar por estar en acuerdo con la estructura usual.

El camino recorrido permitió avanzar en la comprensión de la evaluación de los aprendizajes, así como también habilitó la comprensión de la complejidad que reviste el tema.

Desde este lugar y a partir de los resultados y evidencias obtenidos es posible proponer algunas reflexiones como puntos de interés para futuras líneas de investigación y que puedan ser consideradas como desafíos en nuevas propuestas políticas de educación.

Se plantea de esta forma continuar con la investigación y reflexión acerca de cómo inciden las modalidades tradicionales de evaluación que aún se mantienen en el Instituto de Profesores en algunas de las asignaturas específicas, en las prácticas y en la formación de los futuros profesores. 
Sin demasiados cuestionamientos ni fundamentos sólidos más que la validez que se le otorgó a través del tiempo, la permanencia del modelo tradicional y positivista de evaluación pone en riesgo toda posibilidad de cambio e innovación educativos, ya que se estarían perpetuando en las nuevas generaciones de docentes prácticas de aula y metodologías legitimadas por la formación de grado de los profesores del IPA. Esta situación debería ser contemplada por las futuras políticas educativas y propiciarse modificaciones sustanciales en la profesionalización y actualización de los docentes formadores con el fin de disminuir el peso del factor antigüedad en la formación profesional.

El tiempo de que disponen los docentes configuró un obstáculo relevante en la implementación y seguimiento de las propuestas innovadoras de evaluación. Este aspecto debería ser contemplado en futuras líneas de investigación que abordarán el tema del contrato exclusivo de los docentes como alternativa de desarrollo profesional. De la misma manera, el desafío para las nuevas políticas de formación de profesores es considerar la importancia del tiempo pedagógico, didáctico e institucional para disponer de espacios de trabajo colaborativo, de análisis y de reflexión de la práctica docente.

Asimismo, los nuevos diseños curriculares deberían tener presente este factor y promover que los docentes cuenten con el tiempo necesario para el trabajo sistemático de coordinación de las modalidades de evaluación como forma de estimular la reflexión e impulsar una planificación genuina, consensuada, con propósitos, modalidades e instrumentos que acompañen realmente los principios del paradigma cualitativo y permitan a su vez a los docentes mejorar su desempeño a través de la investigación y el análisis de la validez de las modalidades e instancias de evaluación.

En función de los resultados y conclusiones obtenidos en torno a la transición institucional es posible considerar que los cambios en el proceso de evaluación hacia la formación docente universitaria deben ser parte de una política de gestión de mayor amplitud que contemple no sólo innovaciones en la forma de evaluar y organizar el tiempo pedagógico de los profesores en la institución sino que especialmente apueste a construir una nueva cultura profesional sobre la docencia y la evaluación de los aprendizajes en la educación superior.

\section{Bibliografía}

ADMINISTRACIÓN NACIONAL DE EDUCACIÓN PÚBLICA. CONSEJO DIRECTIVO CENTRAL. (2008). Sistema Único Nacional de Formación Docente 2008. Accedido el 19 de marzo, 2014 desde http://www.cfe.edu.uy/images/stories/pdfs/plan_nacional/sundf_2008.pdf

Álvarez Méndez, J. M. (2001). Evaluar para conocer, examinar para excluir. Madrid: Morata.

Camilloni, A. (2005). De herencias, deudos y legados. Una introducción a las corrientes actuales de la didáctica En: Camilloni, Alicia et al. Corrientes didácticas contemporáneas. Buenos Aires: Paidós.

CONSEJO NACIONAL DE EDUCACIÓN. (1977). Plan para la formación docente. Montevideo.

Denzin, N. \& Lincoln, Y. (2005). Introduction: The Discipline and Practice of Qualitative Research. En: Denzin, N.; Lincoln, Y. (Eds.) "The SAGE handbook of qualitative research" California: Sage Publications. Accedido el 19 de marzo, 2014 desde http://books.google.com.uy/books?hl=es\& Ir=\&id=X85J8ipMpZEC\&oi=fnd\&pg=PR9\&dq=\%E2\%80\%A2\%09DENZIN $,+N .+y+L I N C O L N,+Y$ .++(1994).+Introduction:+Entering+the+field+of+qualitative+research\%E2\%80\%9D\&ots=D1P $\mathrm{dKaP5eq \& sig=up7cKURAjZw2I2DHgOOB6qEsGw0 \# v=onepage \& q \& f=false}$

Diaz Barriga, A. (2000). El examen. Textos para su historia y debate. México: Centro de Estudios sobre la Universidad/Plaza y Valdés. 
House, E. (2000). Evaluación, ética y poder. Madrid: Morata.

Kincheloe, J. (2001). Hacia una revisión crítica del pensamiento docente. Madrid: Octaedro.

Litwin, E. (2005). La evaluación: campo de controversias y paradojas o un nuevo lugar para la buena enseñanza. En: Camilloni, Alicia et al. La evaluación de los aprendizajes en el debate didáctico contemporáneo. Buenos Aires: Paidós.

Perrenoud, P. (2008). La evaluación de los alumnos. De la producción de la excelencia a la regulación de los aprendizajes. Entre dos lógicas. Buenos Aires: Colihue.

Santos Guerra, M. A. (1995). Evaluar es comprender. Buenos Aires: Magisterio del Río de la Plata.

Santos Guerra, M. A. (1998). La evaluación: un proceso de diálogo, comprensión y mejora. Málaga: Aljibe.

Stake, R. (1998). Investigación con estudio de casos. Madrid: Morata. 